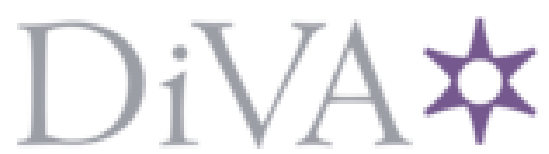

http://www.diva-portal.org

\title{
Postprint
}

This is the accepted version of a paper published in Palliative \& Supportive Care. This paper has been peer-reviewed but does not include the final publisher proof-corrections or journal pagination.

Citation for the original published paper (version of record):

Klarare, A., Rasmussen, B., Fürst, C J., Fossum, B., Lundh Hagelin, C. et al. (2016)

Experiences of security and continuity of care: patients' and families' narratives about the work of specialized palliative home care teams.

Palliative \& Supportive Care

http://dx.doi.org/10.1017/S1478951516000547

Access to the published version may require subscription.

N.B. When citing this work, cite the original published paper.

Permanent link to this version:

http://urn.kb.se/resolve?urn=urn:nbn:se:shh:diva-2310 
Experiences of security and continuity of care - patients' and families' narratives about the work of specialized palliative home care teams. (short title: Security and continuity of care from specialized palliative care teams.)

Anna Klarare - Lecturer, PhD student, MSN, RN. Contact for correspondence.

Sophiahemmet University, Box 5605, 11486 Stockholm, Sweden.

1. Karolinska Institutet, Department of Learning, Informatics, Management and Ethics, Stockholm, Medical Management Centre, Sweden.

2. Sophiahemmet University, Stockholm, Sweden.

Phone: +468 4062955 Mobile: +4676 1856667 Email: anna.klarare@ki.se

Birgit H Rasmussen - Professor, $\mathrm{PhD}, \mathrm{RN}$

1. Lund University, Department of Health Care Sciences, Sweden Institute for Palliative Care Region Skåne and Lund University, Sweden.

Bjöörn Fossum - Professor, PhD, RN

1. Sophiahemmet University, Stockholm, Sweden.

2. Karolinska Institutet, Department of Clinical Science and Education, Södersjukhuset, Stockholm, Sweden.

Carl Johan Fürst - Professor, $\mathrm{PhD}$, MD

1. Institute for Palliative Care Region Skåne and Lund University, Sweden

2. Karolinska Institutet, Department of Oncology-Pathology, Stockholm, Sweden.

Johan Hansson - Researcher, PhD

1. Karolinska Institutet, Department of Learning, Informatics, Management and Ethics, Stockholm, Medical Management Centre, Sweden.

Carina Lundh Hagelin - Senior lecturer, $\mathrm{PhD}, \mathrm{RN}$

1. Sophiahemmet University, Stockholm, Sweden.

2. Stockholms Sjukhem Foundation, Palliative Care Centre, Sweden.

3. Karolinska Institutet, Department of Learning, Informatics, Management and Ethics, Stockholm, Medical Management Centre, Sweden.

Number of manuscript pages: 17

Number of tables: 1

Number of figures: 0 


\section{Experiences of security and continuity of care - patients' and families' narratives about the work of specialized palliative home care teams.}

\section{ABSTRACT}

Being seriously ill and facing death often means living with physical, emotional, social and spiritual suffering. Teamwork is considered to be necessary to holistically meet the diverse needs of persons within palliative care. Reviews of studies regarding palliative care team outcomes conclude that teams provide benefits, especially regarding pain and symptom management. Much of the research concerning palliative care teams has been performed from the perspectives of service providers, and has less often focused on patients' and families' experiences of care. Objective To investigate how the teams' work is manifested in care episodes narrated by patients and families in specialized palliative home care (SPHC).

Methods Thirteen interviews were conducted with patients and families receiving specialized home care. Six patients and seven family members were recruited through specialized palliative home care team leaders. Verbatim interview transcripts were qualitatively analysed into themes. Results Two themes were constructed through thematic analysis: Security: They are always available - I get the help I need quickly and Continuity of care: They know me/us, our whole situation and they really care. In the 74 care episodes, 50 were descriptions of regular, scheduled visits while 24 related to acute care visits and/or interventions.

Significance of results Patients' and family members' descriptions of the work of SPHC teams are conceptualized through experiences of security and continuity of care. Experiences of security are fostered through the 24/7 availability of the SPHC team, sensitivity and flexibility in meeting patients' and families' needs, and practical adjustments to enable care at home. Experiences of 
continuity of care are fostered through the teams' collective approach, where the individual team member knows the patients and families, including their whole situation, and cares about the little things in life as well as caring for the family unit.

Key words: patients, family, health care teams, home care services, palliative care. 


\section{BACKGROUND}

Being seriously ill and facing dying and death means living with the unpredictability of the illness, and experiencing physical, emotional social, and spiritual suffering (Rasmussen et al., 2000; Cassel, 1982) i.e. living with total pain (Clark, 1999). To holistically meet the diverse needs of and to address total pain in persons within palliative care (PC), a specialized PC team is considered to require health care professionals (HCP) with multiple competencies and skills (Junger et al., 2007; Gamondi et al., 2013) for the provision of high quality PC. Knowledge of the effectiveness of PC teams, including specialized palliative home care (SPHC) teams, is gradually accumulating, even if, according to a review by Higginson and Evans (2010), there is still a need to understand which specific components of PC team activity and which types of teams are most effective in achieving desired patient outcomes. Furthermore, much of the research regarding PC teams has been performed either from the perspectives of service providers or on the outcomes of care, often using questionnaire studies, and has less often focused on patients' and families' experiences of being cared for by such a team (Johnston et al., 2012). Terminology regarding PC teams varies, so where previous research is referred to we use the terminology chosen by the original authors.

Reviews of studies investigating outcomes for patients with cancer and their families admitted to specialized PC show positive effects, especially regarding pain and symptom control, and reduction of number of hospital admissions or length of stay (Higginson \& Evans, 2010; Zimmermann et al., 2008). In their retrospective cohort study in Italy, Riolfi et al. (2014) showed that palliative home care services reduced the number of days in hospital in the last two months of life from an average of 20 days to 4 days. Melin-Johansson et al. (2010) explored outcomes by using quality of life assessments in patients with incurable cancer before and after designation to 
a SPHC team, and found significant improvement in global quality of life after inclusion of the team. Another example of outcome focus is found in a 2013 Cochrane review by Gomes et al. (2013a), which concluded that there is clear and reliable evidence that palliative home care teams reduce the symptom burden in patients.

Gomes et al.'s (2013b) review of preferences for care and dying found that most persons prefer to receive home care and to die at home. Palliative home care started in Sweden in the early 1970's. Studies from the past 12 years in Northern Europe investigating patients' and families' experiences of palliative home care have reported that experiencing a sense of security (Goldschmidt et al., 2006; Milberg et al., 2014), accessibility of 24/7 care (Milberg \& Strang, 2011; Hunstad \& Foelsvik Svindseth, 2011), being able to remain at home (Milberg \& Strang, 2007; Appelin \& Bertero, 2004) and experiencing continuity of care (Bostrom et al., 2004; Milberg \& Strang, 2007; Milberg et al., 2012) are key components in the work of the SPHC teams. In 2012, Milberg et al. (Milberg et al., 2012) outlined an overview, a theoretical model, of dying patients' and family members' experiences of palliative home care as a 'secure base'. A sense of security is created, among other things, through trust in the team, being recognized as an individual, experiencing burden relief, being informed and through everyday life at home. This is in line with international findings where patients and family members expressed that the PC team enabled them to cope with difficult circumstances (Noble et al., 2015), that continuity around the clock was paramount (Johnston et al., 2012; Noble et al., 2015; Mehta et al., 2014) and that HCP relations or collaboration were important (Noble et al., 2015; Mehta et al., 2014). Thus, even though benefits of the work of SPHC teams have been identified regarding symptom management, reduced hospital admissions, global quality of life, and through providing a 'secure base', a further exploration is warranted into how persons cared for by the team experience the 
teams' work. This is particularly necessary since replication of qualitative studies is encouraged to "confirm concepts, relationships or patterns" as a strategy to enhance the potential for generalizability (Polit \& Beck, 2010).

Given this background, and in order to explore and strengthen the understanding of which of the components in the teams' work are considered important from the patients' and families' perspectives, our objective was to investigate how the teams' work is manifested in care episodes narrated by patients and families receiving SPHC.

\section{MATERIAL AND METHODS}

\section{Context}

The four SPHC teams providing care to the patients and families included in this study had from 50 to 100 patients in their care at one time, and each team comprised approximately $30 \mathrm{HCPs}$. All teams included physicians, registered nurses (RN), social workers (SW), physical therapists (PT) and occupational therapists (OT) in varying proportions and availability. Team leaders were either physicians or registered nurses. The SPHC team was responsible for all the health care at home, such as symptom management, treatment and nutritional support as well as care regarding social and existential issues, depending on individual situations and needs. The only exception was assistance with, for example, personal hygiene and house cleaning, for which the county home care services were responsible. SPHC teams mainly organize care so that one physician is paired with a group of RNs to constitute the core caring system and so that each patient has a designated RN who is responsible for planning care. The patients in these SPHC units were cared for on average for 58-90 days by the teams. Most HCPs had participated in continuous education 
in PC and, within these teams, three nurses were certified within PC as was one physician. The teams operated in different counties, either in urban or suburban regions, with different reimbursement systems and conditions.

\section{Recruitment and sampling}

Due to the organization of health care services in Sweden and Swedish patient confidentiality laws, SPHC team leaders were asked to independently select and invite patients and families (here persons involved in home care or co-habiting partners) to participate in the study. Brief verbal information about the study was given to prospective participants. If initial consent was given, team leaders provided written information regarding the study and contact information for the research group. The first author contacted potential participants by telephone, re-iterating study information and, if verbal consent was given, an interview was scheduled. All potential patients and family members who gave consent to the team leaders were included in the study.

The inclusion criteria were patients over 18 years of age who were currently enrolled in SPHC or family members of enrolled persons. Information regarding the exact time since enrolment in SPHC was not requested. Exclusion criteria were persons under 18 years of age and persons who did not speak Swedish. During the six-month study period, 13 participants gave consent to be interviewed. The sample consisted of eight women and five men ranging in age from 50 to 89 years. Six patients (three women, three men) with advanced, malignant diagnoses were interviewed, as were seven co-habiting partners (five women, two men), hereafter called family members. These included six patient/family member dyads who were interviewed separately. All participants had completed high school or the equivalent, while four had continued with an associate degree and one with a university degree. 


\section{Data collection}

Data were collected though narrative research interviews (Riessman, 2008b) and inspired by the Critical Incident Technique (CIT) as initially described by Flanagan in 1954 (Flanagan, 1954). After introductory conversations, the starting question was: "Which HCPs do you meet in association with care in your home?". Next, participants were asked to narrate their experiences of specific positive and/or negative care episodes that had occurred. To support participant narratives of the teams' work in the home context, probing questions were used such as: "How did you experience the HCPs' collaboration?” or "Could you tell me more about ...?”, linking to something the participant had mentioned previously if these topics were not addressed spontaneously. Alternatively, echo probing was used, which means repeating the last thing a participant said and asking them to continue. All participants were invited to share one or more care episodes involving HCPs from the SPHC. The interviewer avoided using the English word 'team' since although it is a word used by organizations in Sweden, it is not commonly used and understood in everyday language. The audio-recorded interviews were transcribed verbatim and validated. The tape-recorded part of the interviews, conducted by the first author in the participants' homes, lasted 30-57 minutes (mean 38).

\section{Data analysis}

The study was inspired by the narrative research tradition and thematic analysis (Riessman, 2008b). By performing a thematic analysis, we aimed to remain close to the content of the care episodes narrated by patients and families. The analysis process was conducted in five stages: 
1) The transcripts were checked against the recordings for accuracy. Analysis started with reading the transcribed individual interviews several times to get a sense of the whole.

2) Each interview was read to identify the narrated care situations, i.e. stories of different lengths having a beginning, a middle and an end (Riessman, 2008b), which are from now on called care episodes. These were digitally coloured and inserted into a matrix. For examples of care episodes, see Table 1.

3) Each care episode was analysed and words that the participants used to describe HCPs, such as personal pronouns or names, and words used when evaluating the received care and the encounters with the HCP, were extracted.

4) The thematic analysis of the care episodes continued with highlighting and coding of the content of care episodes, and examining and recording patterns (themes) within and between the care episodes. Patients' and family members' separate narratives of care episodes were compared and, as there were no essential differences in the descriptions of components of the SPHC teams' work, they were analysed together.

5) Finally, the content of the patterns together with the words used to describe and evaluate the components of the SPHC teams' work in the care episodes were discussed, analysed and compared, resulting in two main themes. Inspired by Sandelowski and Leeman (2012), the themes were translated into thematic sentences using participant expressions in an effort to ease understanding of the findings by portraying complete ideas. The quotations from the interviews were translated and pauses, hesitations and repetitions were removed (Riessman, 2008a).

This study received ethical approval from the Regional Ethical Review Committee at Karolinska Institutet (2010/1491-31/3). 


\section{RESULTS}

In total, 74 care episodes involving HCPs in SPHC were narrated by six patients and seven family members. Even though the interviews were rather short, since the patients were severely ill, each patient contributed at least three care episodes. For two examples of care episodes, see Table 1 .

Table 1. Two examples of care episodes in the themes security and continuity of care as narrated by one patient and one family member.

..." this summer I stayed with my sister, I was very wound up and cortisone did not agree very well with me, so I was phasing it out... well anyway, I don't know if it was because of the cortisone or what, but I woke up at 4 or 5 every morning with anxiety. I would call the SPHC... I don't know how many times I called; it was quite a few times... And the person talked to me until I was calm... Getting someone to talk to was enough. Later on I started writing and then I didn't need to call them anymore..." (P3)

"He became really poorly after midsummer... They started giving nutrition at night, ... that started the septicemia..., I guess some nasty stuff got into his blood. And it happened in a few hours, he got a high temperature... but they came straight away and stood here with the doctor on the phone, and it felt like 'yes, they've got it covered'. That felt like WOW... They came for this and supported us and that was great since... It almost caused anxiety before (enrollment in SPHC) to have to call the health care center,... no one (there) has the complete picture, and no one knows us. ... no continuity (P7)

Of the care episodes, 50 were descriptions of regular, scheduled visits while 24 related to acute care visits and/or interventions. Most care episodes narrated by the participants concerned HCPs attending to needs of the whole person, even if the reason behind the contacts, especially in the acute visits, was a physical or pharmacological need. All of the care episodes had positive connotations, although there were a few critical comments, such as a HCP being late or not 
listening to the participant. When talking about the HCPs, participants in 64 of the 74 care episodes used personal pronouns like he, she and they, or the name of the profession like the nurse, the social worker or the physician. In ten episodes personal names were used, like Ruth or Steve. No participants used the word 'team', but in one instance the word 'group' and in two instances the name of the service was used.

The patterns identified in the thematic analysis are presented in the following two condensed themes: Security and Continuity of care. The themes are inseparable entities and not mutually exclusive, therefore care episodes may encompass both themes simultaneously. For clarity, the themes will be presented under separate headings.

\section{Security: They are always available - I get the help I need quickly}

A common thread running through the 37 care episodes in this theme is the participants' experiences of security from being enrolled in SPHC. Patients provided 20 care episodes in this theme and family members provided 17 . Knowing that they can call the HCPs any time, any day, and rely on the fact that a person will answer and do all in their power to help, was significant for experiences of security; see examples in Table 1. Participants did not need to think about whether it was a weekday or weekend, or if it was after business hours, since the same phone number works at all times. A patient put it like this:

"I am really happy and I know that I can call any time and any way, around the clock. There is always someone there to answer." (P1)

Experiences of security not only related to making contact with a HCP, but also to the HCPs sensitivity to changing conditions and circumstances. Team members immediately assessed the 
situation and did what they could to meet these new needs. An example is found in the following quotation:

"A while back I suddenly started vomiting in combination with diarrhoea and it was not long until she (the nurse) was here and listened and I'll be right back she said and then returned with iv fluids... so, in with fluids because I'd lost so much that I was not coherent. Things like that are incredibly valuable, yes, I called..., what do you think I should do? I'll be right over, she said and she came as quickly as she could..." (P8)

Another important aspect of security was experiences of the HCPs adjusting the practical aspects of care to accommodate the patients' and families' situations. In the care episodes, the work of PTs and OTs in modifying the home environment to make home care possible was especially prominent and mentioned by all participants. Many described practical things such as getting a hospital bed delivered and set up, or adjusting bathroom facilities and organizing schedules according to the participant's wishes. A family member stated:

"They have been here and checked what's needed, we have a stair lift and a walker and a toilet seat extension..., we've received incredible help, haven't had to fight for anything, it has come along very smoothly"'(P2)

Other participants told of HCPs finding practical working solutions for the family to enable secure care at home. This entailed assessing the home environment and adapting care provision to suit the environment as stated by a family member: 
"Here they've figured out that it is best to do all the changes and stuff here on the couch. And when we give iv's it's rather convenient to hang the bag from the lamp... so..., all the time they see..., there is lots to use in a home as well and that the people who live there can run things or feel that 'we are a part of this'. That's how they do things. It feels that way anyway..." (P4)

Intertwined with the theme Security is the experience of feeling well cared for. The following quotation from one patient provides the bridge to the theme Continuity of care:

"I am very content because I..., when they leave, they always remind me to call them any time as soon as there is a concern. Well, yes, but I don't want to disturb you... You never disturb us, they say, we are here for you. So, just call us if you need us. Yeah, it's been like that all along and I think that..., I feel privileged and said that to them (the SPHC team)... I feel so well taken care of." (P5)

\section{Continuity of care: They know me/us, our whole situation, and they really care}

Another common thread running through the 37 care episodes in this theme is the participants' descriptions of how both the collaboration between the HCPs and the HCPs approach provide the basis for experiences of continuity of care. This is described both as an experience of a continuous caring relationship, where HCPs know the participants and their whole situation, as well as experiences of the HCPs 'really caring,' even about the 'little things in life'. Patients provided 21 care episodes and family members 16.

The HCPs are described as collaborating, being well informed and knowing the participants. Thus, in contrast to earlier experiences before admission to SPHC, having contact with different 
HCPs is no longer described as problematic. When one HCP leaves the home, the next team member arrives and continues from where the previous HCP left off, without requesting an update from the patient. This does not seem to be linked to particular persons, rather it is demonstrated by all individual HCPs through a united approach in the SPHC team. One patient stated:

..., first I thought that there are so many different persons, but it doesn't matter. They are all like family." (P12).

The different team members merge into a group, or a team, with a mutual approach that includes knowing patients and families. The participants described that even when calling out of office hours, or when a secretary answers the telephone, they just have to say their name and the person at the other end of the line immediately knows who they are, and knows their situation. Even though several HCPs are involved, participants perceive the care they receive as continuous, despite the fact that they may not have met the HCP in person before. A patient expressed it like this:

"The collaboration in this group..., they are tightly knit, they support each other, talk... They quickly tell my story and what kind of person (I am)... So they come here and (say): 'hello hi, so you are David, I almost recognized you even though we've never met', he said..." (P8)

When the HCPs visited and asked questions like, "how was the weekend" or "what are your plans for the holidays", the participants described this interest as an example of caring about the little 
things in life. Other examples of really caring surfaced in care episodes where HCPs followed-up prior issues, even though some time had since passed, as stated by one patient:

"yes, there was a nurse, a man, who came last week and took some blood. And I think he has been here once or maybe twice before. So, he asks me, how is your eating? Because I'd had problems last autumn, I lost a lot of weight... I think it is fantastic that he remembers... They care about the little things, ask how I've been over the holidays, what I've done and so on" (P6)

Being known by the HCPs and experiencing continuity in the care received was a relief for the participants. This is in contrast to their earlier experiences within health care where they had to visit several clinics and explain their situation over and over again to different HCPs. The HCPs are described as managing all the care and having the complete picture of the situation, thus providing care that encompasses more than symptom management. A family member said:

“...It is super with the doctors being so up to speed with everything. They know my husband inside and out, the whole picture that is it... not only symptoms that need curing ... They know exactly, increase a dose or add something or do something else. They really care about him I must say. That is very comforting." (P2)

Narratives illustrate that when a HCP is visiting she/he naturally picks up from where a previous HCP left off, irrespective of the needs that patients and families may have, and also that the HCPs contact each other when a new need arises or when they are made aware of a need that requires a collaborative effort. Also surprising, are the experiences of not only patients and family members 
contacting the SPHC team, but also the other way around. The HCPs contact the patient 'just' to check, for example, if the treatment provided is working. The following quotation is one example:

"well..., I feel like there is collaboration between..., I asked the physiotherapist, I have numb places on my foot that I think are weird, and I asked if there was some kind of exercise or massage that I can do. And as I understand it, the information was relayed to the doctor. That is really good. Also when I've had a common cold, they've checked up on me, been in touch and given me cough syrup etc. And the doctor telephoned me twice just to check up on me..." (P9)

Another important part of the teams' work that the participants described is the feeling of being included, listened to and having the final say in decision-making regarding care. Basing care on the participants' preferences added to experiences of receiving tailored comprehensive care. A patient expressed it like this:

"I make the decisions (regarding care) in collaboration with the SPHC. I might ask questions and I get the answers I need and if they cannot answer, they find out... If they cannot fix it, they come with suggestions, perhaps we can do it this way instead, so... perhaps we can do it another way, to help you with this." (P13)

Likewise, by attending to family needs, the HCPs are described as including and caring for the family members as well as for the patients. For some this was both unexpected and greatly appreciated. The following quotation is one example: 
"they always take a little time to ask and to talk in general about things..., well, with us both, sometimes separately and sometimes together. I think that is lovely, that they ask me too. I am grateful for that..., ...for us and for me in particular, it has been a great comfort." (P1)

In the narrated care episodes, the participants most often used terms like 'we' or 'us', even though one was the patient and the other was not. This is another sign that the teams include the whole family unit in their work. Below is a quote from one family member expressing experiences of feeling cared for:

"Here they know exactly, they take care of and bundle me up in cotton, that's what it feels like, for both of us. And that is exactly what is needed. They are kind and meet us with kindness all the time..." (P10)

The way that the SPHC team works, in particular the attention to detail, results in the entire family experiencing continuity of care. This is an important component of the work of SPHC teams and is exemplified by the caring relationship between the HCPs and the family unit.

\section{DISCUSSION}

The results of the present study strengthen knowledge about important components in the work of the SPHC teams, and provide everyday linguistic expressions of these components from patients' and family members' perspectives. The fostering of experiences of security was accomplished by the 24/7 accessibility of the SPHC team, the quick and adequate responses to problems and the 
mutual approach of collaboration and communication within the team. Intertwined with and in reality inseparable from experiences of security are the experiences of continuity of care. This is shown by all HCPs in the SPHC team knowing the participants and their whole situation, and through their approach, which includes really caring for the whole family unit and also for the little things in life. Interestingly, although the 13 participants came from four different, geographically dispersed, SPHC organizations with different circumstances and systems for reimbursement, patients and families experiences were congruent. Patients' and family members' experiences and descriptions of the work of the SPHC teams in the present study very much resonate with how a palliative care team ought to work (Goldsmith et al., 2010; Parker-Oliver et al., 2005). The results also support Milberg et al.'s (2012) overview of components significant for patients' and families' experiences of security in SPHC.

In this study, as in several other studies (Noble et al., 2015; Milberg et al., 2014; Milberg et al., 2012; Goldschmidt et al., 2006), participants emphasized that experiences of security due to the availability of $24 / 7$ care is a highly relevant feature of the teams' work. Participants in the study described considerable differences from their previous experiences of needing several in-patient, ambulatory care and community health care contacts compared to the present situation with just one phone number to call for all concerns. Despite many different HCPs (in the same service) being involved in the care, the sense of a continuous caring relationship was maintained due to experiences of HCPs collaborating and communicating. The three major attributes of continuity of care described by D'Angelo et al. (2015), namely relationship, communication and comprehensive integrated care, are also described by the participants in this study and resonate with definitions of effective teamwork (Xyrichis \& Ream, 2008; Salas \& Frush, 2013). It seems that the number of HCPs visiting is not key, but rather the atmosphere or approach, which can be 
related for example to team psycho-social traits such as cohesion, norms, efficacy and problemsolving effectiveness as described by Lemieux-Charles and McGuire in the Integrated Health Care Team Effectiveness Model (ITEM) (2006).

Another important component of the teams' work regarding continuity of care for the participants was that they were known by the HCPs. The SPHC team is a group of persons responsible for any concerns or issues regarding care. Even though team members are working shifts and covering all hours, participants state that there is no need to explain, since they are known to the HCPs, as also described by Milberg et al. (2012). Thus experiences of security and continuity of care are in reality intertwined. Regarding organization, Baker, Day and Salas (2006) argue that health care organizations are becoming increasingly dynamic and complex, which results in greater reliance on teams as well as greater complexity with regards to the team's composition and skills. The dynamics of how the SPHC teams manage this remains to be investigated (Higginson \& Evans, 2010; Bosch et al., 2009), especially since team members bring individual knowledge, skills and attitudes (Baker et al., 2006; Salas \& Frush, 2013). In the present study, participants' experienced that the HCPs communicated and collaborated well, even though participants rarely used the word 'team' in reference to HCPs. Irrespective of the individual HCPs, they were all perceived as one, portraying a united approach and even described as family, so that one HCP continued where the previous one left off. This may be contrasted to Mehta et al.'s study (2014) where family members described distress due to lack of information tailored to their families' situations and where individual needs remained unmet. However, a more thorough investigation into the relationship aspect of continuity of care, not necessarily continuity of persons, is an important topic for future research (D'Angelo et al., 2015). 
The word 'team' in patients' and family members' narratives is rarely explicitly stated, as could be expected, and individual HCPs within SPHC are seldom mentioned by name, but are instead described as a group. In the 1990's Tishelman (1994) found that the nurse-patient relationship could be better generalized as a relationship between an individual patient and a collective rather than a one-to-one relationship. Rasmussen and Edvardsson (2007) found that, for hospice patients, the care received as well as the psychosocial and physical environment are inseparable entities, interacting in such a way that it is the "atmosphere of a place" rather than an individual nurse that either supports or hinders patients' experiences of well-being. Interestingly, our findings suggest that not only nurses but the whole interprofessional team appear to be considered as a collective, or family, by patients and families; a collective that together is able to foster an atmosphere of security and experiences of continuity of care. This is in accord with research regarding expert teams that develop a collective way of thinking, feeling and acting (Salas \& Frush, 2013). Health care teams like this are able to shift between being a task-oriented group to being a collective team, in response to changing conditions, without being prompted (Saltman et al., 2007). Findings in the present study suggest that patients and families meet a team, a collective, represented by the individual HCP making the home care visit. These findings add to the growing evidence base regarding important components of security and continuity in SPHC teams' work. However, as most of these findings are based on qualitative research in small samples, an important next step should be a meta-synthesis of research studies on patients' and families' experiences of SPHC teams' work, thereby allowing for more analytic generalizations (Polit \& Beck, 2010). Further research is also needed to better understand the implications of the "collective" approach in SPHC teams. 
There are a number of limitations to be considered in this study. Participants were receiving care from the SPHC team when approached by team leaders and invited to participate, and this may have resulted in a bias towards reporting positive experiences. Participants were interviewed at different time points after the involvement of the SPHC team, which potentially may affect the results depending on the need for care and the illness trajectory. The participants came from urban and suburban areas and the results may therefore not be applicable to rural areas. Other characteristics that may limit transferability were that all participants had at least 9 years of education, with one having received higher education. The participants were all ethnic Swedes and no other ethnic backgrounds were represented. It is possible that persons from other ethnic backgrounds and persons from rural areas would contribute to and develop other important components of SPHC teams' work.

\section{CONCLUSIONS}

Patients' and family members' descriptions of SPHC teams' work is conceptualized through experiences of security and continuity of care. Experiences of security are fostered through the 24/7 availability of the SPHC team, sensitivity and flexibility in meeting patients' and families' needs, as well as practical adjustments being made to enable care at home. Experiences of continuity of care are fostered through the teams' collective approach, where the individual team member knows the patients and families, including their whole situation, and cares about the little things in life as well as caring for the family unit. 


\section{REFERENCES}

Appelin, G. \& Bertero, C. (2004). Patients' experiences of palliative care in the home: a phenomenological study of a Swedish sample. Cancer Nurs, 27(1), 65-70.

Baker, D.P., Day, R. \& Salas, E. (2006). Teamwork as an essential component of high-reliability organizations. Health Serv Res, 41 (4 Pt 2), 1576-1598.

Bosch, M., Faber, M.J., Cruijsberg, J., Voerman, G.E., Leatherman, S., Grol, R.P., Hulscher, M. \& Wensing, M. (2009). Review article: Effectiveness of patient care teams and the role of clinical expertise and coordination: a literature review. Med Care Res Rev, 66(6 Suppl), 5S-35S.

Bostrom, B., Sandh, M., Lundberg, D. \& Fridlund, B. (2004). Cancer patients' experiences of care related to pain management before and after palliative care referral. Eur J Cancer Care (Engl), 13(3), 238245.

Cassel, E.J. (1982). The nature of suffering and the goals of medicine. N Eng/ J Med, 306(11), 639-645.

Clark, D. (1999). 'Total pain', disciplinary power and the body in the work of Cicely Saunders, 1958-1967. Soc Sci Med, 49(6), 727-736.

D'Angelo, D., Mastroianni, C., Hammer, J.M., Piredda, M., Vellone, E., Alvaro, R. \& De Marinis, M.G. (2015). Continuity of care during end of life: an evolutionary concept analysis. Int J Nurs Knowl, 26(2), 80-89.

Flanagan, J.C. (1954). The critical incident technique. Psychol Bull, 51(4), 327-358.

Gamondi, C., Larkin, P. \& Payne, S. (2013). Core competencies in palliative care: an EAPC White Paper on palliative care education - part 1. European Journal of Palliative Care, 20(2), 86-91.

Goldschmidt, D., Schmidt, L., Krasnik, A., Christensen, U. \& Groenvold, M. (2006). Expectations to and evaluation of a palliative home-care team as seen by patients and carers. Support Care Cancer, 14(12), 1232-1240.

Goldsmith, J., Wittenberg-Lyles, E., Rodriguez, D. \& Sanchez-Reilly, S. (2010). Interdisciplinary geriatric and palliative care team narratives: collaboration practices and barriers. Qual Health Res, 20(1), 93-104.

Gomes, B., Calanzani, N., Curiale, V., McCrone, P. \& Higginson, I.J. (2013a). Effectiveness and costeffectiveness of home palliative care services for adults with advanced illness and their caregivers. Cochrane Database Syst Rev, 6, CD007760.

Gomes, B., Calanzani, N., Gysels, M., Hall, S. \& Higginson, I.J. (2013b). Heterogeneity and changes in preferences for dying at home: a systematic review. BMC Palliat Care, 12, 7.

Higginson, I.J. \& Evans, C.J. (2010). What is the evidence that palliative care teams improve outcomes for cancer patients and their families? Cancer J, 16(5), 423-435.

Hunstad, I. \& Foelsvik Svindseth, M. (2011). Challenges in home-based palliative care in Norway: a qualitative study of spouses' experiences. Int J Palliat Nurs, 17(8), 398-404.

Johnston, B.M., Milligan, S., Foster, C. \& Kearney, N. (2012). Self-care and end of life care--patients' and carers' experience a qualitative study utilising serial triangulated interviews. Support Care Cancer, 20(8), 1619-1627.

Junger, S., Pestinger, M., Elsner, F., Krumm, N. \& Radbruch, L. (2007). Criteria for successful multiprofessional cooperation in palliative care teams. Palliat Med, 21(4), 347-354.

Lemieux-Charles, L. \& McGuire, W.L. (2006). What do we know about health care team effectiveness? A review of the literature. Med Care Res Rev, 63(3), 263-300.

Mehta, A., Chan, L.S. \& Cohen, S.R. (2014). Flying blind: sources of distress for family caregivers of palliative cancer patients managing pain at home. J Psychosoc Oncol, 32(1), 94-111. 
Melin-Johansson, C., Axelsson, B., Gaston-Johansson, F. \& Danielson, E. (2010). Significant improvement in quality of life of patients with incurable cancer after designation to a palliative homecare team. Eur J Cancer Care (Engl), 19(2), 243-250.

Milberg, A., Friedrichsen, M., Jakobsson, M., Nilsson, E.C., Niskala, B., Olsson, M., Wahlberg, R. \& Krevers, B. (2014). Patients' sense of security during palliative care-what are the influencing factors? J Pain Symptom Manage, 48(1), 45-55.

Milberg, A. \& Strang, P. (2007). What to do when 'there is nothing more to do'? A study within a salutogenic framework of family members' experience of palliative home care staff. Psychooncology, 16(8), 741-751.

Milberg, A. \& Strang, P. (2011). Protection against perceptions of powerlessness and helplessness during palliative care: the family members' perspective. Palliat Support Care, 9(3), 251-262.

Milberg, A., Wahlberg, R., Jakobsson, M., Olsson, E.C., Olsson, M. \& Friedrichsen, M. (2012). What is a 'secure base' when death is approaching? A study applying attachment theory to adult patients' and family members' experiences of palliative home care. Psychooncology, 21(8), 886-895.

Noble, B., King, N., Woolmore, A., Hughes, P., Winslow, M., Melvin, J., Brooks, J., Bravington, A., Ingleton, C. \& Bath, P.A. (2015). Can comprehensive specialised end-of-life care be provided at home? Lessons from a study of an innovative consultant-led community service in the UK. Eur J Cancer Care (Engl), 24(2), 253-266.

Parker-Oliver, D., Bronstein, L.R. \& Kurzejeski, L. (2005). Examining variables related to successful collaboration on the hospice team. Health Soc Work, 30(4), 279-286.

Polit, D.F. \& Beck, C.T. (2010). Generalization in quantitative and qualitative research: myths and strategies. Int J Nurs Stud, 47(11), 1451-1458.

Rasmussen, B.H. \& Edvardsson, D. (2007). The influence of environment in palliative care: supporting or hindering experiences of 'at-homeness'. Contemp Nurse, 27(1), 119-131.

Rasmussen, B.H., Jansson, L. \& Norberg, A. (2000). Striving for becoming at-home in the midst of dying. Am J Hosp Palliat Care, 17(1), 31-43.

Riessman, C.K. (2008a). Narrative Methods for Human Sciences, Thousand Oaks, California: Sage Publications Inc.

Riessman, C.K. (2008b). Narrative methods for the human sciences, Los Angeles: Sage Publications.

Riolfi, M., Buja, A., Zanardo, C., Marangon, C.F., Manno, P. \& Baldo, V. (2014). Effectiveness of palliative home-care services in reducing hospital admissions and determinants of hospitalization for terminally ill patients followed up by a palliative home-care team: a retrospective cohort study. Palliat Med, 28(5), 403-411.

Salas, E. \& Frush, K. (2013). Improving patient safety through teamwork and team training, New York: Oxford University Press.

Saltman, D.C., O'Dea, N.A., Farmer, J., Veitch, C., Rosen, G. \& Kidd, M.R. (2007). Groups or teams in health care: finding the best fit. J Eval Clin Pract, 13(1), 55-60.

Sandelowski, M. \& Leeman, J. (2012). Writing usable qualitative health research findings. Qual Health Res, 22(10), 1404-1413.

Tishelman, C. (1994). Cancer patients' hopes and expectations of nursing practice in Stockholm--patients' descriptions and nursing discourse. Scand J Caring Sci, 8(4), 213-222.

Xyrichis, A. \& Ream, E. (2008). Teamwork: a concept analysis. J Adv Nurs, 61(2), 232-241.

Zimmermann, C., Riechelmann, R., Krzyzanowska, M., Rodin, G. \& Tannock, I. (2008). Effectiveness of specialized palliative care: a systematic review. JAMA, 299(14), 1698-1709. 